ACCEPTED MANUSCRIPT

\title{
Palmitic acid-coated magnetite nanocubes with high-quality crystallinity and bulk-like magnetic features
}

To cite this article before publication: Juan Manuel Orozco Henao et al 2020 J. Phys. D: Appl. Phys. in press https://doi.org/10.1088/13616463/ab9264

\section{Manuscript version: Accepted Manuscript}

Accepted Manuscript is "the version of the article accepted for publication including all changes made as a result of the peer review process, and which may also include the addition to the article by IOP Publishing of a header, an article ID, a cover sheet and/or an 'Accepted Manuscript' watermark, but excluding any other editing, typesetting or other changes made by IOP Publishing and/or its licensors"

This Accepted Manuscript is @ 2020 IOP Publishing Ltd.

During the embargo period (the 12 month period from the publication of the Version of Record of this article), the Accepted Manuscript is fully protected by copyright and cannot be reused or reposted elsewhere.

As the Version of Record of this article is going to be / has been published on a subscription basis, this Accepted Manuscript is available for reuse under a CC BY-NC-ND 3.0 licence after the 12 month embargo period.

After the embargo period, everyone is permitted to use copy and redistribute this article for non-commercial purposes only, provided that they adhere to all the terms of the licence https://creativecommons.org/licences/by-nc-nd/3.0

Although reasonable endeavours have been taken to obtain all necessary permissions from third parties to include their copyrighted content within this article, their full citation and copyright line may not be present in this Accepted Manuscript version. Before using any content from this article, please refer to the Version of Record on IOPscience once published for full citation and copyright details, as permissions will likely be required. All third party content is fully copyright protected, unless specifically stated otherwise in the figure caption in the Version of Record.

View the article online for updates and enhancements. 


\title{
Palmitic acid-coated magnetite nanocubes with high-quality crystallinity and bulk-like magnetic features
}

\author{
J. M. Orozco-Henao ${ }^{\mathrm{a}}$, D. Muraca ${ }^{\mathrm{b}}$, F. H. Sánchez ${ }^{\mathrm{a}}$, P. Mendoza Zélis ${ }^{\mathrm{a}}$ \\ ${ }^{a}$ Instituto de Física de La Plata (IFLP-CONICET), Departamento de Física, Facultad de \\ Ciencias Exactas, Universidad Nacional de La Plata (UNLP), c.c. 67, 1900 La Plata, \\ Argentina. \\ ${ }^{b}$ Instituto de Física Gleb Wataghin, Universidade Estadual de Campinas (UNICAMP), \\ 13083-970 Campinas, SP, Brasil.
}

\begin{abstract}
We have demonstrated the synthesis of high-quality monocrystalline magnetite nanocubes through the introduction of palmitic acid as surfactant in a thermal decomposition synthesis of an organic $F e$ precursor. Unlike the standard thermal decomposition synthesis route, we report the avoid of the reducing agent and a modification in the synthesis heating ramp. Structural and magnetic properties were investigated showing well defined cubic shaped nanoparticles with a $\sim 40 \mathrm{~nm}$ edge and magnetic features close to bulk magnetite. We associate the bulk-like magnetic performance and properties to the highly crystalline structure of the nanocubes.

In addition, we introduce a facile way to make a ligand exchange of nanocubes initial surfactant to citric acid in order to obtain biocompatible hydrophilic nanocubes. The potential application of the obtained sample in magnetic hyperthermia therapy is shown through calorimetric heating measurements on liquid dispersions of the nanocubes. We compute the Specific Absorption Rate to quantify the heating efficiency of the nanocubes.
\end{abstract}

Keywords: Magnetite nanoparticles, Nanocubes, Magnetic anisotropy, Palmitic acid

\section{Introduction}

Biomedical applications are some of the most promissory uses of magnetic nanoparticles (MNPs) $[1,2,3]$. MNPs based therapies require MNPs colloids with good stability, strong magnetic response to an external field, narrow size distribution and high biocompatibility. For example, the oncology therapy

* Corresponding author

Email address: pmendoza@fisica.unlp.edu.ar (P. Mendoza Zélis) 
6 known as magnetic hyperthermia $(\mathrm{MH})$ is nowadays a trending research. In this 7 therapy, the MNPs are introduced inside the tumor and the region is exposed to a radio frequency field (RF). These MNPs act as conversion agents of the external electromagnetic field into local heat and then release it to their surroundings inducing the apoptosis of malignant cells $[4,5,6]$. The Specific Absorption Rate (SAR), i.e. the amount of power that the particles absorb from the field per unit mass, is an experimental parameter that quantifies this property. The efficiency of the MH treatment depends on the SAR value, which in turn depends on relevant MNPs charactaristics: crystallinity, magnetic anisotropy, magnetic moment, size distribution and geometry.

Iron-oxide nanoparticles (IONPs) synthesis methods have been extensively studied in order to improve their functional properties[7, 8, 9, 10, 11, 12, 13, 14, $15,16,17]$. The chosen synthesis route will determine size distribution, crystallinity degree, magnetic properties, and sample reproducibility. As magnetic properties of IONPs are strongly size-dependent, it is desired to obtain a narrow NPs size distribution in order to tune their response and to improve the NPs performance in the desired biomedical application $[9,2,17,7,18,19,20,21,22]$ Within the various chemical routes of IONPs synthesis, it is well stablished that the thermal decomposition (TD) of organometallic precursors in organic medium allows the production of IONPs with good size control, narrow size distribution, well defined morphology, good crystalline order and relatively easy tunable magnetic features. The main drawbacks of this synthesis method are that some of the reagents commonly used are quite expensive. Also, the obtained NPs are coated with hydrophobic ligands that hinder their biomedical applications. The last issue can be resolved through a ligand exchange process. However, this process could lead to NPs aggregation, NPs surface oxidation and consequently to a lower performance in their application[23].

In this work a new TD type synthesis that produces high-quality singlecrystal nanoparticles (IONPs) showing a well defined cubic shape is reported. Typical TD procedure makes use of oleic acid as the fatty acid surfactant and 1,2-hexadecanediol as reducing agent [24, 25]. Nevertheless, there are various reports on avoiding the reducing agent with no significant modifications in the IONPs magnetic and structural properties[26, 27, 28]. This new TD synthesis follows a similar idea to that reported by P. Guardia et al. [28], introducing palmitic acid (PA, $\left.\left(\mathrm{C}_{16} \mathrm{H}_{32} \mathrm{O}_{2}\right)\right)$ as the fatty acid surfactant, avoiding the use of 1,2-hexadecanediol as reducing agent and modifying the heating ramp, in order to obtain high-quality monocrystalline magnetite nanocubes (IONCs), improv4 ing its magnetic properties and lowering the synthesis costs. Although there are (a few) reports on palmitic acid coating in iron oxide nanoparticles, there is no report (as far as we know) of iron oxide nanocubes with structural and magnetic properties as the ones we present here from TD synthesis and PA surfactant. ${ }_{47}$ For example, Sawisai et al. report the synthesis of iron oxide nanoparticles by 48 co-precipitation and a posterior palmitic acid coating process[29]; Bronstein et $49 a l$. , the production of IONPs from a thermal decomposition synthesis of iron 50 palmitate as precursor[30]; Klekotka et al. reports a thermal decomposition synthesis from Fe(III)acac, phenyl ether and 1,2-hexadecanediol[31]. 
To provide the hydrophilic character we propose and successfully performed 53 a ligand exchange of palmitic acid to citric acid (CA) by means of a mechano54 chemical route. Ligand-exchange processes in equivalent situations have been 55 widely reported elsewhere. Nevertheless, the process described here constitutes 56 a facile and effective one-step process to produce iron oxide NCs, which does 57 not require further use of catalyst reagents. To our knowledge, this synthesis 58 procedure has not been reported before. We have tested if this ligand exchange induces any alteration of the NP properties and on its SAR performance.

The experimental results show that both NCs samples are high-quality singlecrystals and single-domain showing almost bulk magnetic features. From electron microscopy and Mössbauer spectroscopy it was possible to quantify and determine the effective anisotropy constant of the samples. Also, it was corroborated the high magnetic saturation of the NCs as a consequence of a high quality monocrystalline structure. It was confirmed that the ligand exchange process did not modify neither the NCs structural nor magnetic properties. In 7 addition, the potential application of the obtained system in magnetic hyperthermia treatment was tested through the evaluation of heat dissipation per9 formance under an external RF field for two different dispersion mediums, e.g. distilled water and toluene. Owing to the cubic morphology of the NPs, a tendency to form NCs chains was expected and corroborated by TEM and SAXS 72 experiment. This feature is known that leads to an improvement of the heating ${ }_{3}$ dissipation performance[32, 33, 21]

\section{Experimental section}

\section{2.1. Synthesis}

Magnetite nanocubes were synthesized by means of the thermal decomposition of $2 \mathrm{mmol}$ of $\mathrm{Fe}(I I I)_{\text {acac }}$ (Sigma Aldrich), $8 \mathrm{mmol}$ of palmitic acid (Merck) and $50 \mathrm{~mL}$ of Benzylether (98\%, Sigma Aldrich) in a three-neck flask. The mixture was heated, under $N_{2}$ atmosphere and vigorous magnetic stirring, from room temperature to reflux at a rate of $3^{\circ} \mathrm{C} / \mathrm{min}$. The solution was kept under reflux temperature for $30 \mathrm{~min}$ and then cooled down to RT. $30 \mathrm{~mL}$ of ethanol was added to stabilize the final solution. The product was washed several times with ethanol and centrifuged many times, in order to remove the PA excess. The obtained precipitate was dried in vacuum to obtain the PA coated IONCs powder sample. From now on this sample will be labeled as $S_{T}$. Two more samples where obtained following the same synthesis process showing a high reproducibility in structural and magnetic properties (see supplementary information).

\subsection{Ligand exchange}

$2 \mathrm{~mL}$ of a $10 \mathrm{mg} / \mathrm{mL}$ NCs suspension in toluene were mixed with $200 \mathrm{mg}$ of citric acid monohydrate (Anedra) and $10 \mathrm{~mL}$ of distilled water. The solution was put under sonication for $1 \mathrm{~h}$, then placed for $48 \mathrm{~h}$ in a Retsch 2000 oscillatory mill under a low energy regime ( $5 \mathrm{~Hz}$ and $8 \mathrm{~mm}$ of amplitude). The final product 
was washed several times with toluene and then centrifuged in order to remove the PA and CA excess. Finally, the black precipitate was vacuum dried. From now on this sample will be labeled as $S_{W}$.

\subsubsection{HR-TEM Images}

HR-TEM images of the NCs were obtained using a TEM-FEG (TALOS F200A) and a TEM-FEG (JEM 2100F) field-emission gun transmission electron microscopes. First, the particles were dispersed in toluene and sonicated for 15 minutes, then, the samples for microscopy observation were prepared by drying a drop of this suspension during 24 hours at room temperature on a Ted Pella ultrathin copper film on a holey carbon grid. The obtained images were analyzed using Digital Micrograph free version software ${ }^{1}$.

\subsection{2. $S A X S$}

SAXS measurements were carried out at the Instituto de Investigaciones Fsicoqumicas Tericas y Aplicadas (INIFTA) facilities in a XENOCS XEUSS 1.0 diffractometer with a beam wavelength of $1.5419 \AA$. The samples where placed in a glass capillary at $2.4 \mathrm{~m}$ distance from detector. The results correspond to the SAXS intensity as a function of the scattering vector for each dispersion of $10 \mathrm{mg}$ of the sample in $1 \mathrm{~mL}$ of toluene.

\subsubsection{Mössbauer spectroscopy}

The $14.4 \mathrm{keV}\left(I g=1 / 2 ; M 1 ; I_{e}=3 / 2\right)^{57} \mathrm{Fe}$ Mössbauer spectra were acquired using a ${ }^{57} \mathrm{Co}(\mathrm{Rh})$ source at $295 \mathrm{~K}$ in transmission geometry, with a standard spectrometer operating in the constant acceleration regime. The isomer shifts are reported relative to $\alpha$-Fe. Each sample absorber thickness was 12 $\mathrm{mg} / \mathrm{cm}^{2}$. The number of channels used for recording transmitted gamma versus source-absorbent velocity was 1024 . The spectrometer line width was 0.22 $\mathrm{mm} / \mathrm{s}$ for a $12 \mu \mathrm{m}$-foil $\alpha$-Fe absorber.

\subsubsection{D.C Magnetization measurements}

The magnetic properties were studied by means of thermal dependence of magnetization through zero field cooled/field cooled $(z f c / f c)$ and isothermal field dependence of magnetization measurements. $z f c / f c$ experiments were performed in a MPMS XL superconducting quantum interference device (SQUID) from Quantum Design, Inc. In the $z f c$ magnetization measurement, the sample was cooled down to $4 \mathrm{~K}$ from RT under no applied field, then a $4 \mathrm{kA} / \mathrm{m}$ (50 Oe) field was applied and the temperature was continuously raised at a $5 \mathrm{~K} / \mathrm{min}$ rate while recording the magnetization. In the $f c$ magnetization measurement, the sample was cooled down to $4 \mathrm{~K}$ from $\mathrm{RT}$ at a $5 \mathrm{~K} / \mathrm{min}$ rate under a 4 $\mathrm{kA} / \mathrm{m}(50 \mathrm{Oe})$ applied field while recording the magnetization. Additional $z f c$ magnetization measurements on sample $S_{W}$, using three different applied fields $(4 \mathrm{kA} / \mathrm{m}, 8 \mathrm{kA} / \mathrm{m}$ and $40 \mathrm{kA} / \mathrm{m})$, were performed.

${ }^{1}$ http://www.gatan.com/products/tem-analysis/gatan-microscopy-suite-software 
Isothermal field dependent magnetization loops were measured in a vibrating sample magnetometer (VSM) at RT within an applied magnetic field strength $\mu_{0} H$ range of $-2 \mathrm{~T}$ to $2 \mathrm{~T}$. All d.c. magnetization measurements were carried out using $5 \mathrm{mg}$ of sample powder compacted in thin gelatin diamagnetic capsules. Magnetizations values are reported in terms of iron-oxide mass determined by thermogravimetry analysis.

\subsubsection{Experimental SAR values}

The heating performance of both samples was evaluated measuring the Specific Absorption Rate (SAR). This parameter is related to the amount of energy absorbed per time unit and per mass unit of IONPs exposed to a radio frequency field (RF). This energy is then released to their surroundings as heat. For each sample, SAR values were obtained from calorimetric measurements carried out in two colloidal suspensions of $10 \mathrm{mg}$ of NCs sample in $1 \mathrm{~mL}$ of toluene and distilled water for samples $S_{T}$ and $S_{W}$, respectively, held in a clear glass Dewar. An external RF of $52 \mathrm{kA} / \mathrm{m}$ amplitude and $260 \mathrm{kHz}$ frequency was applied. The field generator (Hüttinger $(2.5 / 300)$ ) consists of a resonant RLC circuit with a water refrigerated 6 turns coil of $2.5 \mathrm{~cm}$ inner diameter. The temperature $T$ of each colloid as a function of time $t$ was measured. Temperature was sensed during the experiment with an optical fiber provided with a small semiconducting sensor at one end, which was placed at the sample center. The sensor was connected to a calibrated signal conditioner (Neoptix) having an accuracy of $\pm 0.1^{\circ} \mathrm{C}$. The colloid temperature was kept below $50^{\circ} \mathrm{C}$ in order to minimize solvent evaporation and prevent its destabilization. Each of the reported values is a 4 measurements mean with an error equal to the standard deviation SD. SAR was calculated as

$$
S A R=\frac{\rho c}{[C]} \frac{\Delta T}{\Delta t}[W / g]
$$

where $\rho$ is the suspension density taken as the toluene or distilled water density in $\mathrm{kg} \cdot \mathrm{m}^{-3}$ for $S_{T}$ and $S_{W}$, respectively, $c$ is the dispersive medium heat capacity in $\mathrm{J} \cdot \mathrm{g}^{-1} \cdot \mathrm{K}^{-1}, \Delta T / \Delta t$ is the $T(t)$ slope (at $T$ equals to the lab temperature) and $[C]$ is the colloid weight concentration of iron-oxide mass in $\mathrm{kg} \cdot \mathrm{m}^{-3}$.

\subsubsection{Thermogravimetry analysis}

A Thermogravimetry (TG) analysis was used to estimate the iron-oxide fraction mass of the coated NPs. The measurements were carried out on dried powder samples with Shimatzu TG-50 and DTA-50 systems. During the measurements the samples were kept inside platinum crucibles and heated at a 10 $\mathrm{K} / \mathrm{min}$ constant rate under a $20 \mathrm{~mL} / \mathrm{min} N_{2}$ flux. It was further used to normalize magnetization measurements and to express colloid concentrations as the mass of iron oxide per toluene/distilled water volume. Results are presented in the supplementary information. 


\section{Results and discussion}

\subsubsection{TEM and HR-TEM images}

Morphology, particle-size distributions, and crystalline phases were determined by means of electron microscopy experiments. Figure 1 shows representative TEM images and the size distribution obtained from several similar images of samples $S_{T}$ (a,b) and $S_{W}$ (d,e). Well defined cubic shaped particles with mean edge sizes $\left(L_{0}\right), L_{0_{S_{T}}}=39.7 \mathrm{~nm}, L_{0_{S_{W}}}=36.7 \mathrm{~nm}$, with standard deviations $\sigma_{S_{T}, S_{W}}=0.2$, were obtained by counting of more than $300 \mathrm{NPs}$ for each sample. To quantify these statistics estimators, a Log-Normal distribution was fitted to the measured size distribution histograms and is shown for each sample in figure $1(\mathrm{c}, \mathrm{f})$.

Figure 2 shows a HR-TEM image of isolated groups of NCs of both samples, the (400) and (220) planes of the FCC structure are highlighted with red/blue and green colors, respectively. To obtain the colorful images, first a fast Fourier transformation (FFT) was applied to the initial HR-TEM image (figure 2 (a) and (d)) to obtain a profile of the diffraction patterns (figure 2 (b) and (e)) allowing us to identify the NCs crystallographic planes; then a mask was applied to distinguish each plane in the original image and finally an inverse FFT was made over each mask in order to label planes with the colors already mentioned (figure 2 (c) and (f)). Identification of crystallographic planes allowed us to obtain a mean lattice parameter of 0.85 Afor both samples. The NCs show a high crystallinity degree with the magnetite lattice planes growth facet $\{100\}$. This result is in agreement with other magnetite nanocubes systems reported in previous works $[34,35,36]$. A correlation of these structural features with magnetic properties is reported in other works that conclude that these well defined lattice planes on the NCs contribute to an increment on individual surface anisotropy and so to the effective anisotropy of the system [37, 32].

From HR-TEM images and the subsequent FFT analysis it can be concluded that synthesized NCs are single-crystalline with narrow size distribution and are also within the single-domain limit for magnetite[38, 39]. Furthermore, one can see that there must be an ordered attachment mechanism in which the NCs clusters are formed, with a clear trend to a chain like structure.

\subsection{2. $S A X S$}

SAXS measurements bver liquid dispersions were measured on both samples in order to obtain information about nanoparticles morphology, agglomeration degree and cluster geometry. SAXS intensity patterns were successfully fitted by considering a cubic form factor along with a Log-Normal size distribution of the NCs edges. Clusters formation due to interparticle interactions were taken into account by means of a mass fractal structure factor. On the other hand, the scattering intensity contribution from NCs that do not form clusters were modeled by considering local interparticle interactions (excluded volume) in first approximation by a hard spheres interaction potential. Figure 3 shows experimental SAXS patterns and the corresponding fit for both samples. At low scattering vector $(q)$ values, SAXS patterns are consistent with the existence of 


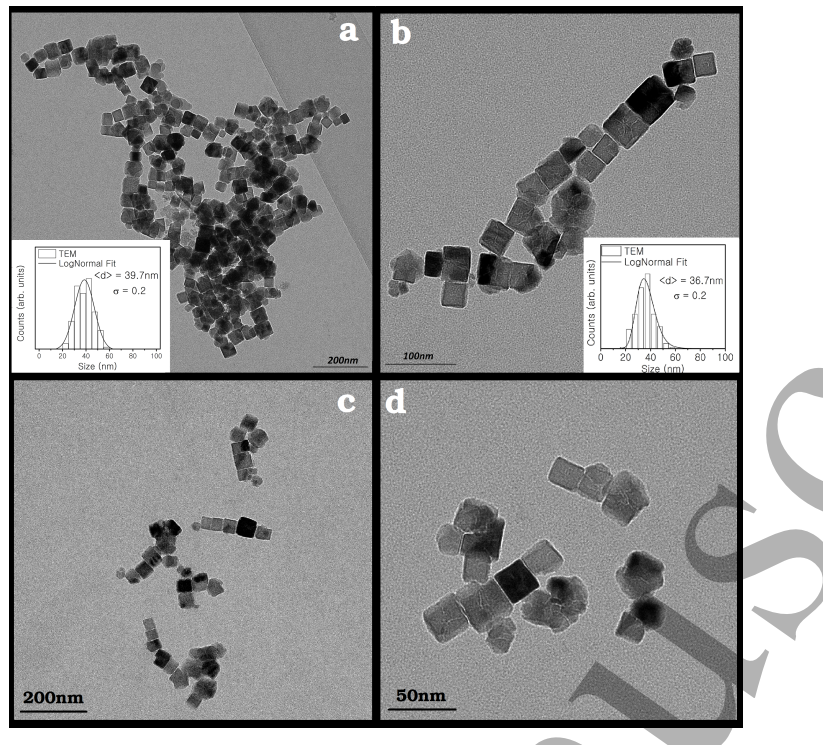

Figure 1: TEM images and size distribution histograms fitted with a Log-Normal function for the studied samples. Sample $S_{T}(\mathrm{a}, \mathrm{c})$ and sample $S_{W}(\mathrm{~b}, \mathrm{~d})$.

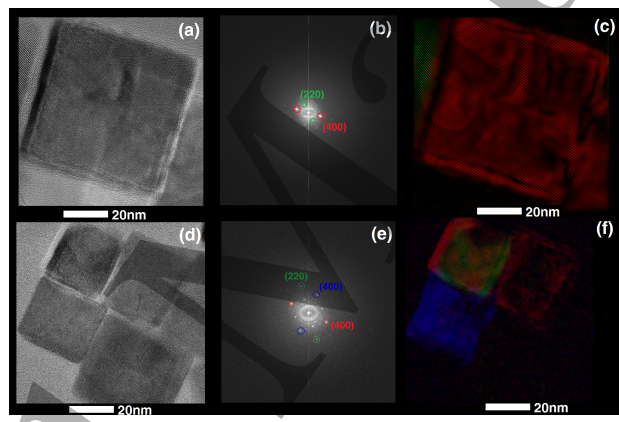

Figure 2: (a) and (d): HR-TEM images of nanocubes. (b) and (e): Fourier transform of the initial HR-TEM images. (c) and (f): colored images obtained from an inverse process masking the identified diffraction points.

215 aggregates of NCs. For $q$ values near $0.2 \mathrm{~nm}^{-1}$ the patterns show a bump related 216 to the NCs mean size.

To fit the experimental data, the cubic form factor is given by $[40,41]$

$I(q, R)=\iint_{0}^{\pi / 2}\left[\rho V \frac{\sin \left(\frac{q R}{2} \sin \theta \sin \phi\right)}{\frac{q R}{2} \sin \theta \sin \phi} \frac{\sin \left(\frac{q R}{2} \sin \theta \cos \phi\right)}{\frac{q R}{2} \sin \theta \cos \phi} \frac{\sin \left(\frac{q R}{2} \cos \theta\right)}{\frac{q R}{2} \cos \theta}\right]^{2} \sin \theta \mathrm{d} \theta \mathrm{d} \phi$

where $\rho$ is the scattering contrast of the NPs relative to the medium and $R$ is the cube edge. 
The mass fractal model for NP aggregates follows a power law [42, 43]. Here $\xi$ stands for the upper cut-off of clusters size.

$$
S_{m f}(q, \xi)=1+\frac{1}{(q R)^{D}} \frac{D \Gamma(D-1)}{\frac{1}{2}(D-1)} \frac{\sin [(D-1) \arctan (q \xi)]}{\left(1+(q \xi)^{-2}\right)}
$$

On the other hand, the hard sphere structure factor $S_{H S}(q, r)$ with the Percus-Yevick closure[44, 45] is given by:

$$
S_{h s}(q, r)=\frac{1}{1+24 f_{p} \frac{G\left(f_{p}, q r\right)}{q r}}
$$

where $f_{p}$ is the local volume fraction of particles within the clusters. This allowed us to get information about the probability of finding NCs in the vicinity of each other. Here,

$G\left(f_{p}, q r\right)=\alpha \frac{\sin A-A \cos A}{A^{2}}+\beta \frac{2 A \sin A+\left(2-A^{2}\right) \cos A-2}{A^{3}}+\gamma \frac{-A^{4} \cos A+4\left[\left(3 A^{2}-6\right) \cos A+\left(A^{3}-6 A\right) \sin A+6\right]}{A^{5}}$

with $A=2 q r, \alpha=\left(1+2 f_{p}\right)^{2} /\left(1-2 f_{p}\right)^{4}, \beta=-6 f_{p}\left(1+f_{p} / 2\right)^{2} /\left(1-f_{p}\right)^{4}$ and $\gamma=\alpha f_{p} / 2$.

The polidispersity nature of the NCs was modeled by including a Log-Normal distribution function $L(R, \sigma)$ of their edges. The final distributed intensity function takes the form:

$I_{d}(q, R)=N 1\left[\int_{0}^{\infty} I(q, R) L(R, \sigma) S_{m f}(q, R) \mathrm{d} R\right]+N 2\left[\int_{0}^{\infty} I(q, R) L(R, \sigma) S_{h s}(q, R) \mathrm{d} R\right]+b k g$

where $N 1$ and $N 2$ are the Log-Normal distribution weights and are relative to the NCs number per mass unit for each contribution (mass fractal and hard spheres potential). $b k g$ is a constant added to take into account the incoherent background contribution.

Figure 3 inset shows the excellent agreement between the NCs size distribution obtained from HR-TEM images and the one obtained from SAXS measurements. Table 1 shows the fitting parameters obtained for both studied samples. Both Log-Normal distributions have similar parameters $\sigma$ and $L_{0}$ values. The volume fraction $f_{p}$ values close to 0.5 indicates that NCs tend to form clusters in both colloids. Cubic nanoparticles in colloids present an extraordinary magnetic self-assembly capability as reported in the works of Singh et al. and Mehdizadeh et al.[47, 48]. The self-assembly nature of the nanocubes allows the formation of various types of structures. For low concentration colloids, 1-D chain formation is preferred. This chain formation of clusters were observed by TEM images where a low concentration colloid was used. At higher concentrations, chain-chain association assemblies become more stable leading to the formation of 2D sheets[48]. In this sense, a fractal dimension close to 2 indicates that the cluster structure tends to define a surface, in concordance with the higher 
concentration used to perform SAXS measurements. Although a direct correspondence between structures and colloid concentrations cannot be stated by SAXS and TEM without the aid of other structural characterization techniques, the structures observed seem to show coherence with the expected results.

The upper cut-off of cluster size $\xi$ was fixed to $114 \mathrm{~nm}$ which is the lowest possible value congruent with the lowest $q$ measured value by means of the relation $2 \pi / \xi=0.04 \mathrm{~nm}^{-1}[46]$.

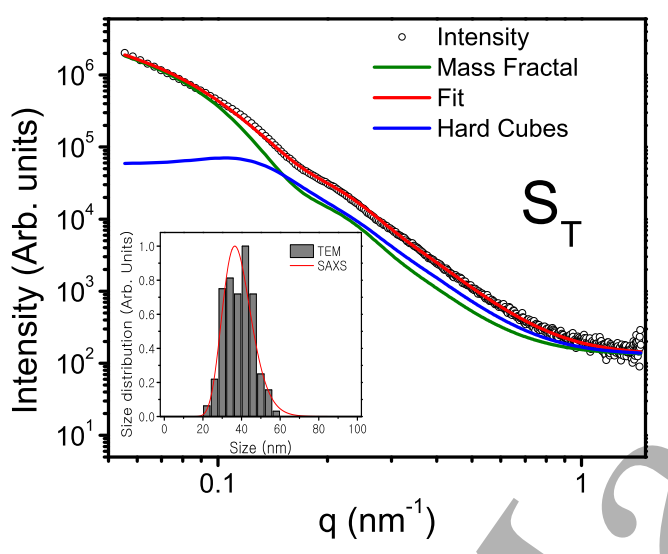

(a)

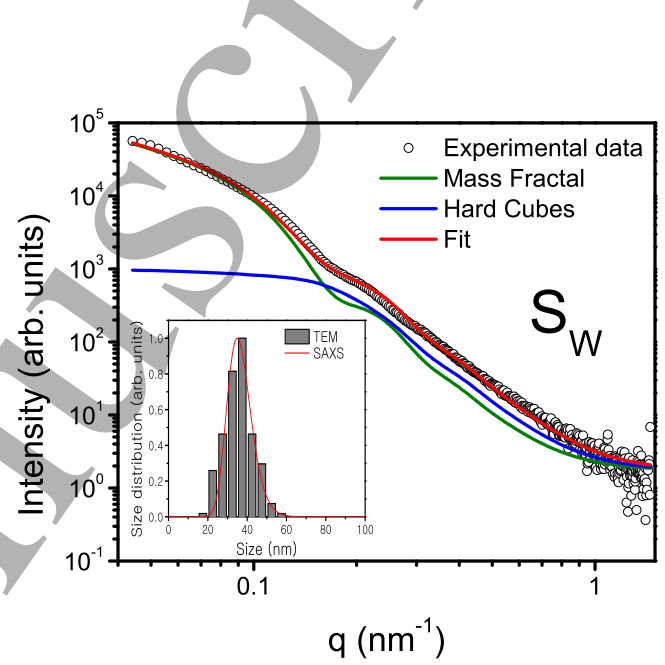

(b)

Figure 3: SAXS intensity pattern and corresponding fit for sample (a) $S_{T}$ and (b) $S_{W}$. Inset shows the agreement between TEM and SAXS analysis.

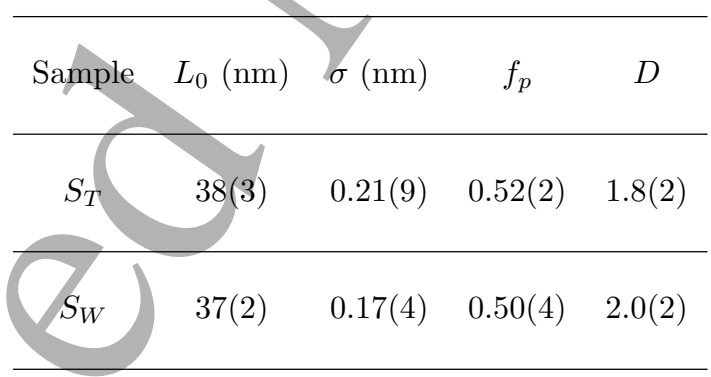

Table 1: SAXS parameters. Mean particle edge $L_{0}$, standard deviation $\sigma(\mathrm{nm})$, local volume fraction of particles $f_{p}$ and fractal dimension $D$.

251 We have shown so far the structural properties of IONCs obtained by TD 252 synthesis of an organic precursor of $\mathrm{Fe}$. We also have successfully incorporated 253 palmitic acid as the IONCs surfactant. The synthesis route introduced in this 254 work avoids the use of 1,2-hexadecanediol as reducing agent and incorporates $\mathrm{PA}$ as the NCs surfactant. PA is a fatty acid with a 16 carbon atoms chain 
and a boiling point of $\sim 350^{\circ} \mathrm{C}$ with a good solubility in benzyl-ether. These

properties are quite similar to those of oleic acid (18 carbon atoms and boiling point of $360^{\circ} \mathrm{C}$ ) with the particular difference that PA does not present a double bond in its carbon chain. Owing these similarities between the two fatty acids we can assume that the temperature regimes of nucleation and growing phase during the synthesis[24, 49] are nearly the same in both cases. However, the shape and size of the obtained NPs depends on the fatty acid selected for the synthesis. When oleic acid is used small NPs $(5-20 \mathrm{~nm})$ with almost spherical shape are obtained [24, 49, 25], whereas when we use palmitic acid, NPs with a well-defined cubic shape $(\sim 40 \mathrm{~nm})$ are obtained. This fact reveals that nanoparticle formation dynamics is different in both cases.

From the structural properties results it can be concluded that the election of PA as surfactant leads to the production of high-quality monocrystalline NCs by means of a highly reproducible and low cost synthesis route. These structural features are in agreement with the results from the synthesis reported by Guardia et al.[28], in which the use of 1,2-hexadecanediol is avoided and decanoic acid (10 single-bonded carbon chain) is chosen as the fatty acid surfactant.

\subsubsection{Mössbauer spectroscopy}

The magnetization dynamics and magnetic response of MNPs magnetic moment is given by the Néel and/or Brown relaxation mechanisms. The later is due to the random physical rotation of MNPs in a liquid medium and is absent during Mössbauer measurements of powder samples. The Néel mechanism is due to the rotation of the magnetic moment within the particle. Because of the MNPs magnetic anisotropy, the magnetic moment has equilibrium orientations separated by energy barriers. At finite temperature, there is a finite probability for the magnetic moment to change its direction. The mean time between two direction changes is called the Néel relaxation time $\tau_{N}$. It depends on the NP energy barriers created by the magnetic anisotropy.

A relation between $\tau_{N}$ and the characteristic time of a magnetic measurement $\left(\tau_{m}\right)$ will determine the observed magnetic dynamics of the system. If $\tau_{m}>\tau_{N}$ then the observed properties will correspond to a superparamagnetic system (thermodynamic equilibrium); if $\tau_{m}<\tau_{N}$ the observed properties will correspond to a magnetically "blocked" system. As $\tau_{N}$ depends on temperature, the blocking temperature $T_{B}$ is defined as the temperature that divides these two observed magnetic regimes. Below $T_{B}$ the MNPs are magnetically blocked; for temperatures above $T_{B}$ the MNPs are in thermodynamic equilibrium. Thus, the blocking temperature is related to the time window of the experimental measurement. In particular, for Mössbauer spectroscopy $\tau_{m} \sim 10^{-8} \mathrm{~s}$.

If we consider a particle in its blocked state, its magnetic moment orientation will be confined around one of its equilibrium positions, but fluctuating about an easy magnetization direction. The fluctuation time of the magnetic moment within the equilibrium well is short compared with the Mössbauer spectroscopy time scale, so the hyperfine parameters will be affected by this phenomena. The magnetic splitting of the observed Mössbauer spectra, and so the measured 
hyperfine field $\left(H_{o b s}\right)$, will be related to the mean value of the system hyperfine field in the energy well[50]

$$
H_{o b s}=H_{0} \iint_{\Omega} P\left(u_{x}, u_{y}\right) u_{z} \mathrm{~d} u_{x} \mathrm{~d} u_{y}
$$

where $\left(u_{x}, u_{y}, u_{z}\right)$ are the direction cosines of the magnetization vector. Here we take $u_{z}$ as the easy direction. $P\left(u_{x}, u_{y}\right)$ is the orientation probability of the magnetization vector and $\Omega$ defines the limits of the potential well:

$$
P\left(u_{x}, u_{y}\right)=\frac{\exp \left[-E\left(u_{x}, u_{y}\right) / k_{B} T\right]}{\iint_{\Omega} \exp \left[-E\left(u_{x}, u_{y}\right) / k_{B} T\right] \mathrm{d} u_{x} \mathrm{~d} u_{y}}
$$

Here $k_{B}$ is the Boltzmann constant and $T$ the absolute temperature. Integration in equation (6) is solved by taking into account that below $T_{B}$ the magnetization vector fluctuates around small values of $u_{x}$ and $u_{y}$, by considering that the anisotropy energy is higher than the thermal energy, so one obtains:

$$
H_{o b s} / H_{0} \approx 1-\frac{1}{2} k_{B} T\left[\left(\frac{\partial^{2} E}{\partial u_{x}^{2}}\right)_{0}^{-1}+\left(\frac{\partial^{2} E}{\partial u_{y}^{2}}\right)_{0}^{-1}\right]
$$

which can be written as

$$
H_{o b s} / H_{0}=1-\frac{k_{B} T}{K_{e f f} V} \quad ; \quad K_{e f f} \equiv 2\left[\left(\frac{\partial^{2} E}{\partial u_{x}^{2}}\right)_{0}^{-1}+\left(\frac{\partial^{2} E}{\partial u_{y}^{2}}\right)_{0}^{-1}\right]^{-1}
$$

where $K_{\text {eff }}$ is the effective anisotropy constant and $V$ the NP volume[51, 50].

Mössbauer spectrum was analyzed considering two magnetically split subspectra, each one consistent of six absorption lines. Figure 4 shows the Mössbauer spectrum of sample $S_{T}$ together with the fitted curve and each sub-spectra. One of the sextets represents the absorption due to $\mathrm{Fe}^{3+}$ ions in tetrahedral sites $\left(\mathrm{Fe}_{A}^{3+}\right)$. The other sextet accounts for absorption due to $\mathrm{Fe}^{2.5+}$ ions in octahedral sites $\left(\mathrm{Fe}_{B}^{2.5+}\right)$. The nomenclature $2.5+$ stands for the fast electron hopping between $2+$ and $3+$ ions at octahedral locations[52]. The fitting model allows for deviations from magnetite standard stoichiometry. Distribution of the hyperfine interactions associated with NCs volume distribution was also incorporated in the fitting model. The volume distribution was assumed to be Log-Normal. We used equation (9) in order to link both types of distributions. Mean hyperfine parameters obtained from model are summarized in table 2 from which the presence of magnetite can be identified.

Isomer shift and hyperfine field values obtained from fit are in good agreement with those for non-stoichiometric magnetite[53]. Accordingly to Cheng[54] and $\mathrm{Ho}[34]$, the outermost layers composed of octahedral sites are relatively more stable than those composed of tetrahedral sites, at the $\mathrm{Fe}_{3} \mathrm{O}_{4}\{001\}$ surface. In this way, the terminated $\{001\}$ planes are mostly composed of octahedral $\mathrm{Fe}^{2+}, \mathrm{Fe}^{3+}$ and oxygen ions, increasing the $\mathrm{Fe}_{B}^{2.5+} / \mathrm{Fe}_{A}^{3+}$ ratio. 


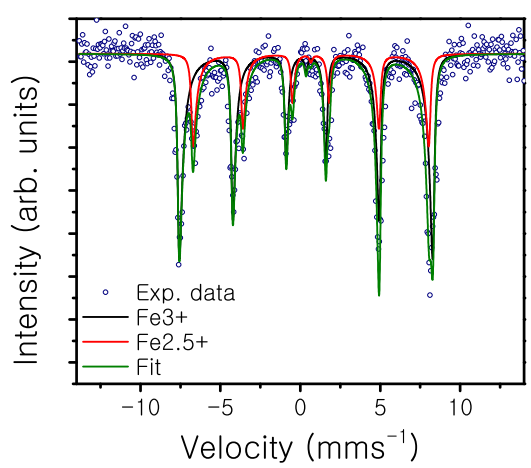

(a)

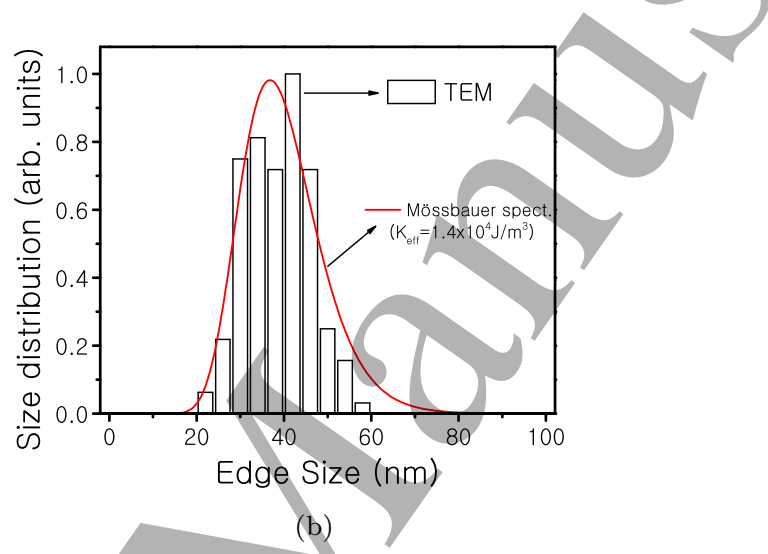

Figure 4: (a) Mössbauer spectrum, experimental data and fit (continuous line) at RT. (b) Comparison of NCs size distribution obtained from HR-TEM results and from hyperfine field distribution analysis. Effective anisotropy constant derived from Mössbauer spectrum was $K_{\text {eff }}=1.4 \times 10^{4} \mathrm{~J} / \mathrm{m}^{3}$.

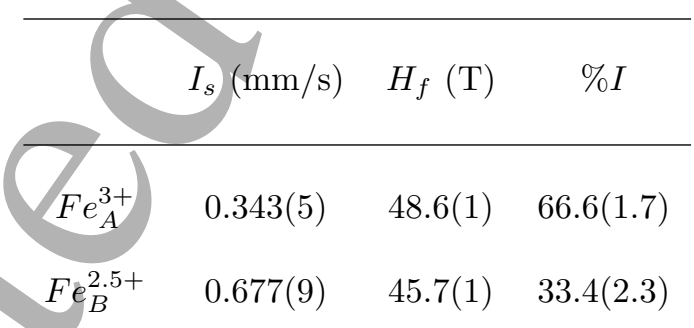

Table 2: The mean values of the hyperfine parameters obtained from the analysisi of figure 4(a): isomer shift $\left(I_{s}\right)$, Hyperfine field $\left(H_{f}\right)$ and Area relations $(\% I)$. A null quadrupolar splitting was obtained. 
Additional information about the stoichiometry of the sample is obtained by considering the mean-isomer-shift method[55, 56]: the area-weighted meanisomer-shift at RT $\overline{I S}_{R T}$ is correlated with the magnetite/maghemite sample composition, and allows to determine the sample stoichiometry. The $\overline{I S}_{R T}$ parameter shows a linear correlation with $\alpha$, being $\alpha$ the atomic percentage of $\mathrm{Fe}$ atoms present in the form of magnetite in the mixture[56]. We have computed the $\overline{I S}_{R T}$ by: a) direct determination $\overline{I S}_{R T}=\Sigma[($ Background Count $\left.\left._{i}\right) V l_{i}\right] / \Sigma\left[\left(\right.\right.$ Background-Counts $\left.\left._{i}\right)\right]$ were Count $_{i}$ is the number of counts of chanel $i$ and $\mathrm{Vel}_{i}$ the source velocity associated to this chanel, b) fit procedure suggested by Fock et al. [56], and c) from our fitting model. The $\overline{I S}_{R T}$ value obtained by the three methods coincide and is $\overline{I S}_{R T}=0.45 \pm 0.02$, which corresponds to $\alpha=0.63 \pm 0.08$.

A deviation from stoichiometry has been evaluated through the general formula $\mathrm{Fe}_{3(1-\epsilon)} \mathrm{O}_{4}$ using the relation $\epsilon=(1-\alpha) /(9-\alpha)[56]$. In our case, the nanocubes correspond to magnetite with a slight deviation from stoichiometry as $\mathrm{Fe}_{2.87} \mathrm{O} 4$ with $\epsilon=0.044$. It is worth to say that there is no evidence of a maghemite phase contribution from the Mössbauer spectrum. This departure from the pure magnetite stoichiometry is reflected in some magnetic properties as it will be shown in the following sections.

From Mössbauer spectrum fit by means of equation (9), parameters of the Log-Normal distribution of NCs volumes are obtained considering a constant $K_{\text {eff }}$ in the product $K_{e f f} V$. The comparison with the size distribution obtained from HR-TEM can be used to determine an effective mean $K_{\text {eff }}$ value as the one that maximizes the coincidence between TEM and Mössbauer NCs size distributions. A value of $1.4(1) \times 10^{4} \mathrm{~J} / \mathrm{m}^{3}$ leads to a very good correspondence between TEM and Mössbauer data (see figure 4(b)). The measured effective anisotropy must be compared to the theoretical value, calculated with the second expression of (9), which for a cubic system with easy axes in the $\{111\}$ directions equals to $-4 / 3 K_{1}-4 / 9 K_{2}[50]$. For bulk magnetite at RT[58] $K_{1}=-1.2 \times 10^{4}$ $\mathrm{J} / \mathrm{m}^{3}$ and $K_{2}=-0.3 \times 10^{4} \mathrm{~J} / \mathrm{m}^{3}$, resulting in $K_{\text {eff }}=1.7 \times 10^{4} \mathrm{~J} / \mathrm{m}^{3}$.

The experimental result is $17 \%$ smaller than this value, most probably due to the departure from pure magnetite stoichiometry and surface magnetic disorder. Effective anisotropy constant slightly lower than the bulk one was already reported by Ludwing et al. [59] $\left(1.2 \times 10^{4} \mathrm{~J} / \mathrm{m}^{3}\right)$ and Bender et al.[60] $\left(1.7 \times 10^{4} \mathrm{~J} / \mathrm{m}^{3}\right)$ both for magnetite spheres with $19 \mathrm{~nm}$ diameter.

Other anisotropy sources, from surface, shape and dipolar interactions between particles, are involved in the estimation of the effective anisotropy constant. In the special ease of NCs, magnetostatic energy is isotropic for an uniformly magnetized cube[61], so it will not modify magnetization anisotropy. On the other hand, no field was applied during Mössbauer experiments, hence non local effects of dipolar interactions can be neglected. It is worth recalling that Mössbauer experiments performed on blocked NPs under no applied field are affected by collective fluctuations of magnetic moments around a local energy minimum. Although these are far from surpassing the anisotropy energy maxima, differences between $K_{\text {eff }}$ values determined with this technique and with magnetometry methods can be expected. 
It is remarkable how, an appropriate combination of TEM and Mössbauer results, leads to a consistent description of anisotropy and size distribution properties.

\subsection{4. $Z F C / F C$ magnetization curves}

Figure 5 shows the $z f c / f c$ magnetization results for both $S_{T}$ and $S_{W}$ samples. A qualitative analysis shows that both samples are in average in the blocked regime over the whole range of studied temperatures, no global blocking temperature is observed over this range of temperature. Also, the irreversibility temperature, i.e. the temperature above which the system loses its magnetic memory, is above RT. The $f c$ curve shows an almost constant behavior during all the measurement process unveiling strong interparticle interactions. The $z f c$ curves show distinctive features in the low temperatures range[62]. To highlight these features with more detail, $d M_{z f c} / d T(T)$ was plotted in figure 5 insets. The maximum of $d M_{z f c} / d T(T)$ around $30 \mathrm{~K}$ in sample $S_{T}$ could be due to a surface phenomenon originated by the dislocation of spins laying on the NCs surface[63, 64].

The $S_{W}$ sample has similar magnetic properties as sample $S_{T}$. However, it shows small differences in the magnetization magnitude and its behavior at low temperatures. For example, the $f c$ magnetization is $\sim 2 \mathrm{Am}^{2} / \mathrm{kg}$ less than that of the $S_{T}$ sample and the maximum of the $d M_{z f c} / d T(T)$, which appears at around $30 \mathrm{~K}$ for $S_{T}$ is at $15 \mathrm{~K}$ for $S_{W}$. This is mainly related to the ligand exchange process and to its influence on the atoms at the NCs surface $[65,66,67]$.

Although presenting similar structural and magnetic properties, sample $S_{W}$ has a better defined $d M_{z f_{c}} / d T$ profile than sample $S_{T}$. Figure 6 shows in detail the behavior of the function $d M_{z f c} / d T$ for three different applied fields on sample $S_{W}$. It can be noticed that the temperature at which both maxima occur $(15 \mathrm{~K}$ and $85 \mathrm{~K})$ does not depend on the intensity of the applied magnetic field. In consequence, these two effects are not thermally activated supporting that the effect that appears at $15 \mathrm{~K}$ should be due to surface phenomena. The maximum at $85 \mathrm{~K}$ could be attributed to the Verwey transition[68], in agreement with the result reported by Muscas[62]. This transition is expected at $120 \mathrm{~K}$ for bulk magnetite. However, lower/values have been reported and attributed to size and stoichiometric effects $[69,57,70,71]$. Anyhow, the presence of the Verwey transition, also noticeable after the ligand exchange process, confirms the presence of magnetite with a high crystalline ordering[72].

\subsubsection{Isothermal magnetization curves}

Figure 7 shows the field dependent isothermal magnetization loop at RT for both samples; inset shows magnetization behavior in the low field range. $S_{T}$ and $S_{W}$ samples showed a coercive field of $6.0(7) \mathrm{kA} / \mathrm{m}$ and $5.4(6) \mathrm{kA} / \mathrm{m}$, respectively, revealing that the system is on average in the blocked regime at room temperature. Saturation Magnetization $\left(M_{s}\right)$ was evaluated by fitting the magnetization (high-field region) versus $1 / \mathrm{H}$ according to the Law of Approach to Saturation[73]. Obtained $M_{s}$ values were 82(1) $\mathrm{Am}^{2} / \mathrm{kg}$ and 84(1) $\mathrm{Am}^{2} / \mathrm{kg}$ for $S_{T}$ and $S_{W}$ samples, respectively. 


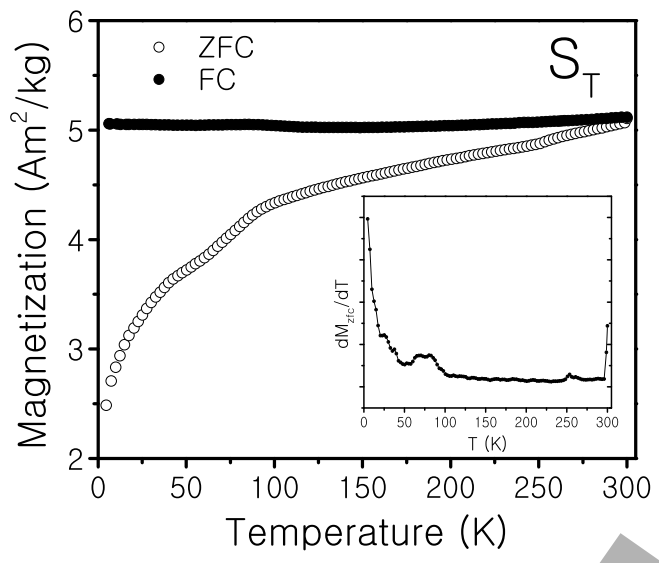

(a)

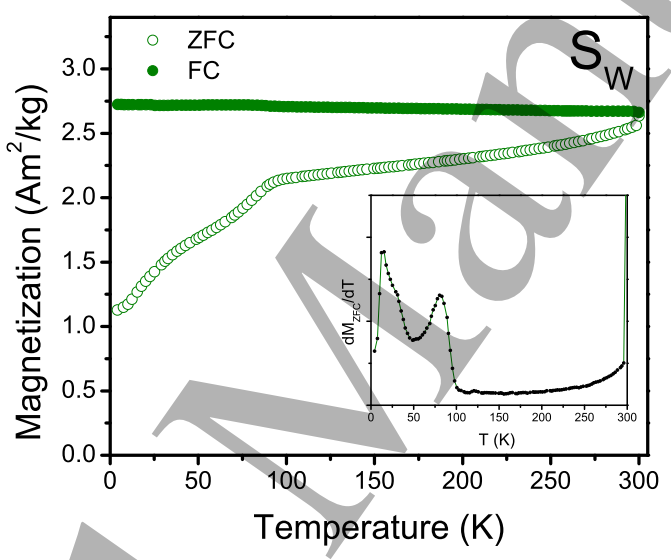

(b)

Figure 5: Zero field cooled and field cooled magnetization of (a) sample $S_{T}$ and (b) sample $S_{W}$. Inset shows $d M_{z f_{c}} / d T(T)$ as function of temperature.

As it was mentioned, from the Mossbauer measurements the magnetite stoichiometry was determined. To study the effects of this deviation from stoichiometry on magnetic properties like the magnetic saturation it is important to know both the amount of $F e$ atoms in tetrahedral and octahedral sites and its oxidation state. In this sense it may be preferable to report the formula unit in a form that highlights the oxidative transformation[55]: $F e^{3+}\left[F e_{a}^{2+} F e_{a}^{3+} F e_{b}^{3+} \square_{2-2 a-b}\right] O_{4}$. In our case, the values obtained from $\alpha$ are $a=0.60$ and $b=0.66[56]$. The number of Böhr magnetons $\left(\mu_{B}\right)$ per unit formula can be ealculated as $5(b+a-1)+4 a$, considering the antiparallel arrangement of magnetic moment between tetrahedral and octahedral sites, and 


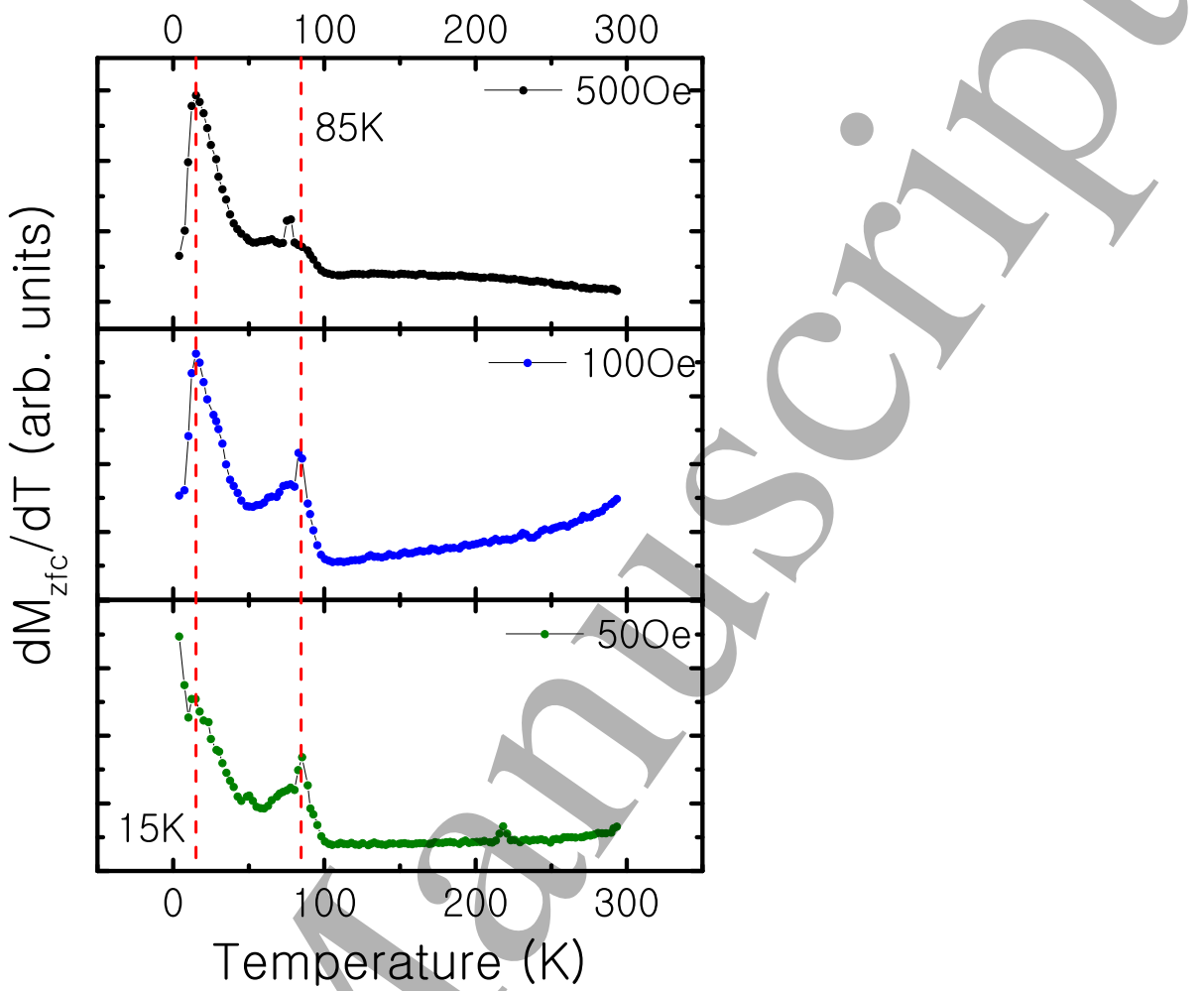

Figure 6: $d M_{z f c} / d T$ temperature behavior for three different applied fields on sample $S_{W}$.

that $F e^{2+}$ and $F e^{3+}$ have $4 \mu_{B}$ and $5 \mu_{B}$, respectively. The value obtained is $3.7 \mu_{B}$. This reduction, in comparison to the $4 \mu_{B}$ expected for pure magnetite, is in very good agreement with the reduction in the saturation magnetization measured in our sample $(82 \mathrm{emu} / \mathrm{g})$ in comparison with the expected for pure magnetite (92 emu/g)

The high coercive field at RT may be due to dipolar interactions between particles and/or strong spin interactions given the high crystallinity order of the NCs[39]. It is well known that the NPs structural properties affect the system magnetic properties. In this sense, the high mean magnetic moment and saturation magnetization obtained in our NCs is due to their high quality monocrystalline structure [72]. As in $z f c / f c$ magnetization curves, the small difference between $S_{T}$ and $S_{W}$ saturation magnetization values is mostly due to the magnetic disorder created by dislocated surface spins after the ligand exchange process $[70,28,74,63]$. 
Figure 7: Field dependent magnetization loop at room temperature. Fitted curves are shown as continuous lines for each sample. Inset shows magnetization behavior in the low applied fields zone.

\subsubsection{Power dissipation}

Figure 8 shows the heating curves for samples $S_{T}$ and $S_{W}$, respectively, under an RF field of amplitude $H=57 \mathrm{kA} / \mathrm{m}$ and frequency $f=260 \mathrm{kHz}$.Power dissipation values of 324(9) W/g and a 304(7) W/g were computed from experimental data by means of equation (1). Our SAR results are in good agreement with those reported for IONPs systems with similar structural and magnetic properties[77, 32, 75, 76]. The NP cubic morphology plays a key role on magnetic hyperthermia performance due mostly to an increment on individual NP magnetic anisotropy and the ease of the NCs to self-assemble in chains. This feature results in an enhancement of the coercive field due dipolar interactions and thus, a better heating performance [32, 33, 21]. The chain formation degree and so the dissipation capacity can be tailored by controlling the NCs concentration, therefore increasing or reducing the interparticle interactions as reported by Coral et al. [78]. Besides this, magnetic nanoparticles in a colloid may relax by either of two mechanisms being Néel and Brownian relaxation. However, in the actual therapy, the particles end mostly aggregated and fixed into cellular structures; therefore the Browns dissipation mechanism is canceled and the SAR value could change. Also, it was suggested that the principal effect of the internalization of MNPs by living cells is due the increase in agglomeration (and in dipolar interactions) rather than immobilization[79, 80].

From the obtained results, it is also clear that the ligand exchange process 


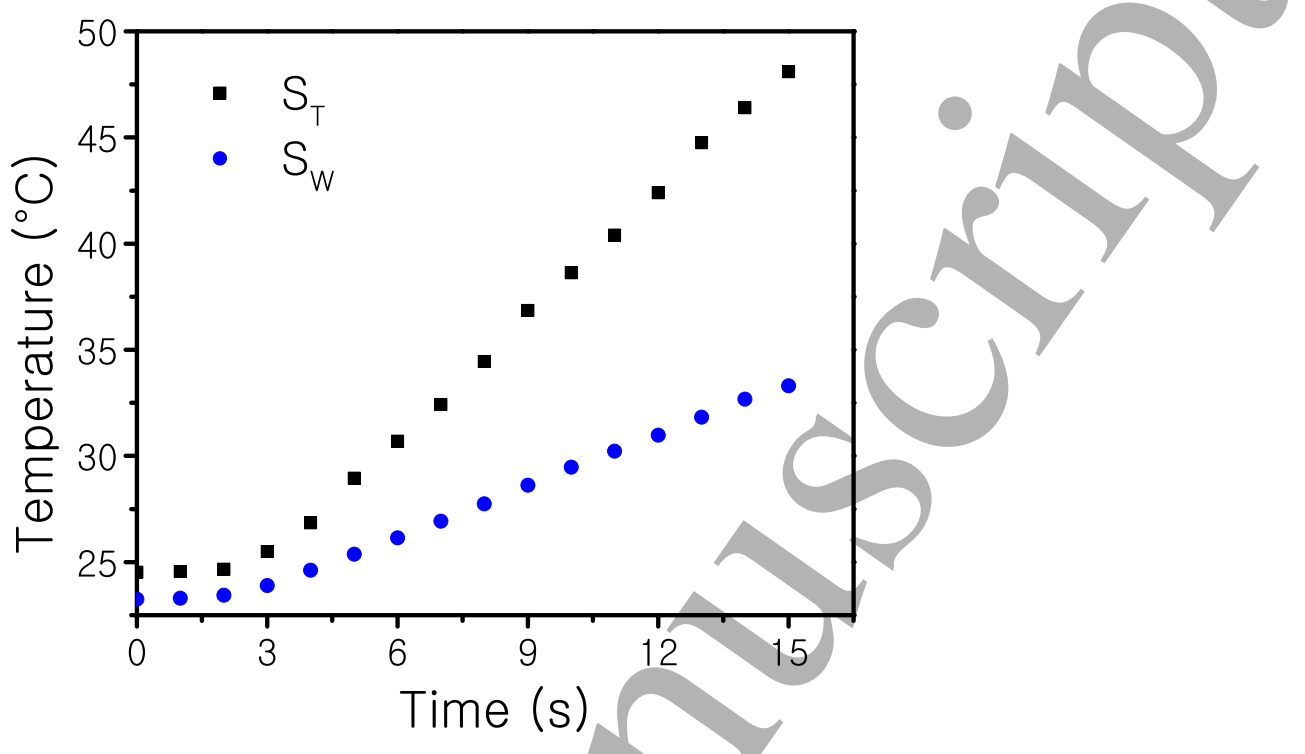

Figure 8: Heating rate curve of NCs suspensions in toluene (sample $S_{T}$ ) and distilled water (sample $S_{W}$ ). Magnetic field amplitude $H=52 \mathrm{kA} / \mathrm{m}$ and frequency $f=260 \mathrm{kHz}$.

does not modify the heating performance of the $S_{T}$ sample. The slope difference between both measurements lies on differences in medium density and heat capacity.

\section{CONCLUSIONS}

In this work, we described the structural and magnetic properties of a system of cubic IONPs. These IONPs, which have a $\sim 40 \mathrm{~nm}$ edge, were obtained by thermal decomposition synthesis, and we have introduced palmitic acid as a novel MNPs surfactant. We have developed a synthesis protocol with promising results on fundamental research and magneto-hyperthermia application.

The set of structural and magnetic properties determined in this study demonstrates that we have obtained high quality MNPs with well-defined cubic shape and high crystallinity degree. The after-synthesis ligand-exchange process described here is an easy way to overcome the hydrophobic character of the synthesized sample. We want to highlight the fact that the structural and magnetic properties of the initial sample were not modified after this procedure.

Due to its heat dissipation capacity, quantitatively characterized by the SAR value, the studied IONCs sample can be considered as a good candidate for magnetic hyperthermia treatment. The obtained SAR and ILP values are comparable with previously reported results on similar IONPs systems. The heating efficiency of the presented system appears to be enhanced by the NPs cubic 
shape and their capability of forming chain-like clusters, compared with other NPs samples with similar structural properties reported elsewhere.

We want to emphasize that the low-cost synthesis method that we have developed keeps, or even enhances, the desirable system properties for the abovementioned applications. It is also worth highlighting that the synthesis results are highly reproducible, allowing the preparation of NPs systems with the same specific structural and magnetic properties.

\section{ACKNOWLEDGMENTS}

We acknowledge CONICET and UNLP of Argentina for financial support through Grant Nos. PIP 0720 and X11/680. We also want to aknowledge YPF Tecnologa (Y-TEC) and Ph.D Alberto Caneiro for HR-TEM images, Instituto de Investigaciones Fisicoqumicas Teóricas y Aplicadas (INIFTA) and Ph.D Lisandro Giovanetti for SAXS measurements. D.M. acknowledges Brazilian Nanotechnology National Laboratory (LNNano) at Centro Nacional de Pesquisa em Energia e Materiais (CNPEM)/MCTI for the use of electron microscopy facility (proj. number 22452, 22424), FAPESP (Fundação de Amparo á Pesquisa do Estado de São Paulo grant 2017/10581-1), National Council for Scientific and Technological Development (CNPq, 303236/2017-5). We want to thank Ph.D Pablo Tancredi from Universidad de Buenos Aires (UBA) for synthesis process discussions and advice.

\section{References}

[1] T. Neuberger, B. Schöpf, H. Hofmann, M. Hofmann, B. Von Rechenberg, Superparamagnetic nanoparticles for biomedical applications: Possibilities and limitations of a new drug delivery system, J. Magn. Magn. Mater. 293 (2005) 483-496.

[2] Q. A. Pankhurst, N. T. K. Thanh, S. K. Jones, J. Dobson, Progress in applications of magnetic nanoparticles in biomedicine, J. Phys. D. Appl. Phys. 42 (2009) 224001.

[3] M. L. Tebaldi, C. M. Oda, L. O. Monteiro, A. L. de Barros, C. J. Santos, D. C. F. Soares, Biomedical nanoparticle carriers with combined thermal and magnetic response: Current preclinical investigations, J. Magn. Magn. Mater. 461 (2018) 116-127.

[4] M. H. Falk, R. D. Issels, Hyperthermia in oncology, Int. J. Hyperth. 17 (2001) 1-18.

[5] R. E. Rosensweig, Heating magnetic fluid with alternating magnetic field, 522 J. Magn. Magn. Mater. 252 (2002) 370-374.

[6] S. Dutz, R. Hergt, Magnetic particle hyperthermia - A promising tumour therapy?, Nanotechnology 25 (2014). 
[7] N. Lee, T. Hyeon, Designed synthesis of uniformly sized iron oxide nanoparticles for efficient magnetic resonance imaging contrast agents, Chem. Soc. Rev. 41 (2012) 2575-2589.

[8] M. Brollo, J. Orozco-Henao, R. López-Ruiz, D. Muraca, C. Dias, K.Pirota,

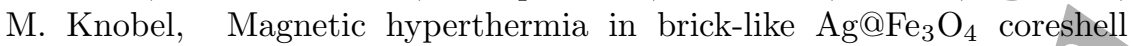
nanoparticles, J. Magn. Magn. Mater. 397 (2015) 20-27.

[9] A. K. Gupta, M. Gupta, Synthesis and surface engineering of iron oxide nanoparticles for biomedical applications, Biomaterials 26 (2005) 3995 4021.

[10] O. Moscoso-Londoño, J. S. Gonzalez, D. Muraca, C. E. Hoppe, V. A. Alvarez, A. López-Quintela, L. M. Socolovsky, K. R. Pirota, Structural and magnetic behavior of ferrogels obtained by freezing thawing of polyvinyl alcohol/poly(acrylic acid) (PAA)-coated iron oxide nanoparticles, Eur. Polym. J. 49 (2013) 279-289.

[11] P. Tancredi, S. Botasini, O. Moscoso-Londoño, E. Méndez, L. Socolovsky, Polymer-assisted size control of water-dispersible iron oxide nanoparticles in range between 15 and 100nm, Colloids Surfaces A/Physicochem. Eng. Asp. 464 (2014) 46-51.

[12] Y. Lu, Y. Yin, B. T. Mayers, Y. Xia, Modifying the Surface Properties of Superparamagnetic Iron Oxide Nanoparticles through a Sol-Gel Approach, Nano Lett. 2 (2002) 183-186.

[13] P. Mendoza Zélis, D. Muraca, J. S. Gonźalez, G. A. Pasquevich, V. A. Alvarez, K. R. Pirota, F. H. Sánchez, Magnetic properties study of ironoxide nanoparticles/PVA ferrogels with potential biomedical applications, J. Nanoparticle Res. 15 (2013).

[14] S. Laurent, D. Forge, M. Port, a, Roch, C. Robic, L. V. Elst, R. N. Muller, Magnetic Iron Oxide Nanoparticles: Synthesis, Stabilization, Vectorization, Physicochemical Characterizations, and Biological Applications (vol 108, pg 2064, 2008), Chem. Rev. 108 (2008) 2064-2110.

[15] S. Ge, X. Shi, K. Sun, C. Li, C. Uher, J. R. Baker, M. M. Banaszak Holl, B. G. Orr, Facile Hydrothermal Synthesis of Iron Oxide Nanoparticles with Tunable Magnetic Properties, J. Phys. Chem. C 113 (2009) 13593-13599.

[16] M. Mikhaylova, D. K. Kim, N. Bobrysheva, M. Osmolowsky, V. Semenov, T. Tsakalakos, M. Muhammed, Superparamagnetism of Magnetite Nanoparticles: Dependence on Surface Modification, Langmuir 20 (2004) 2472-2477.

[17] R. M. Fergusón, K. R. Minard, A. P. Khandhar, K. M. Krishnan, Optimizing magnetite nanoparticles for mass sensitivity in magnetic particle imaging, Med. Phys. 38 (2011) 1619-1626. 
[18] G. Salas, C. Casado, F. J. Teran, R. Miranda, C. J. Serna, M. P. Morales, Controlled synthesis of uniform magnetite nanocrystals with high-quality properties for biomedical applications, J. Mater. Chem. 22 (2012) 21065.

[19] B. Issa, I. Obaidat, B. Albiss, Y. Haik, Magnetic Nanoparticles: Surface Effects and Properties Related to Biomedicine Applications, Int. J. Mol. Sci. 14 (2013) 21266-21305.

[20] L. Gutiérrez, R. Costo, C. Grüttner, F. Westphal, N. Gehrke, D. Heinke, A. Fornara, Q. A. Pankhurst, C. Johansson, M. P. Morales, Synthesis methods to prepare single- and multi-core iron oxide nanoparticles for biomedical applications, Dalt. Trans. (2015) 2943-2952.

[21] M. Cho, A. Cervadoro, M. Ramirez, C. Stigliano, A. Brazdeikis, V. Colvin, P. Civera, J. Key, P. Decuzzi, Assembly of Iron Oxide Nanocubes for Enhanced Cancer Hyperthermia and Magnetic Resonance Imaging, Nanomaterials 7 (2017) 72 .

[22] J. Panda, B. S. Satapathy, S. Majumder, R. Sarkar, B. Mukherjee, B. Tudu, Engineered polymeric iron oxide nanoparticles as potential drug carrier for targeted delivery of docetaxel to breast cancer cells, J. Magn. Magn. Mater. 485 (2019) 165-173.

[23] N. Jović Orsini, B. Babić-Stojić, V. Spasojević, M. P. Calatayud, N. Cvjetićanin, G. F. Goya, Magnetic and power absorption measurements on iron oxide nanoparticles synthesized by thermal decomposition of $\mathrm{Fe}(\mathrm{acac})_{3}$, J. Magn. Magn. Mater. 449 (2018) 286-296.

[24] S. Sun, H. Zeng, Size-controlled synthesis of magnetite nanoparticles, J. Am. Chem. Soc. $124(2002) 8204-8205$.

[25] J. M. Orozco-Henao, D. F. Coral, D . Muraca, O. Moscoso-Londoño, P. Mendoza Zélis, M. B. Fernández Van Raap, S. K. Sharma, K. R. Pirota, M. Knobel, Effects of Nanostructure and Dipolar Interactions on Magnetohyperthermia in Iron Oxide Nanoparticles, J. Phys. Chem. C 120 (2016) 1279612809.

[26] Y. Hou, Z. Xu, S. Sun, Controlled synthesis and chemical conversions of FeO nanoparticles, Angew. Chemie - Int. Ed. 46 (2007) 6329-6332.

[27] Z. Xu, C. Shen, Y. Hou, H. Gao, S. Sun, Oleylamine as both reducing agent and stabilizer in a facile synthesis of magnetite nanoparticles, Chem. Mater. 21 (2009) 1778-1780.

[28] P. Guardia, A. Labarta, X. Batlle, Tuning the Size, the Shape, and the Magnetic Properties of Iron Oxide Nanoparticles, J. Phys. Chem. C 115 (2011) $390-396$. 
[29] R. Sawisai, R. Wanchanthuek, W. Radchatawedchakoon, U. Sakee, Simple continuous flow synthesis of linoleic and palmitic acid-coated magnetite nanoparticles, Surfaces and Interfaces 17 (2019) 100344.

[30] L. M. Bronstein, J. E. Atkinson, A. G. Malyutin, F. Kidwai, B. D. Stein, D. G. Morgan, J. M. Perry, J. A. Karty, Nanoparticles by decomposition of long chain iron carboxylates: From spheres to stars and cubes, Langmuir 27 (2011) 3044-3050.

[31] U. Klekotka, D. Satuła, S. Spassov, B. Kalska-Szostko, Surfactant dependence on physicochemical properties of magnetite nanoparticles, Colloids Surfaces A Physicochem. Eng. Asp. 537 (2018) 452-459.

[32] C. Martinez-Boubeta, K. Simeonidis, A. Makridis, M. Angelakeris, O. Iglesias, P. Guardia, A. Cabot, L. Yedra, S. Estradé, F. Peiró, Z. Saghi, P. A. Midgley, I. Conde-Leborán, D. Serantes, D. Baldomir, Learning from nature to improve the heat generation of iron-oxide nanoparticles for magnetic hyperthermia applications, Sci. Rep. 3 (2013) 1-8.

[33] E. C. Abenojar, S. Wickramasinghe, J. Bas-Concepcion, A. C. S. Samia, Structural effects on the magnetic hyperthermia properties of iron oxide nanoparticles, Prog. Nat. Sci. Mater. Int. 26 (2016) 440-448.

[34] C. H. Ho, C. P. Tsai, C. C. Chung, C. Y. Tsai, F. R. Chen, H. J. Lin, C. H. Lai, Shape-controlled growth and shape-dependent cation site occupancy of monodisperse Fe3O4nanoparticles, Chem. Mater. 23 (2011) 1753-1760.

[35] M. E. Sadat, R. Patel, J. Sookoor, S. L. Bud'Ko, R. C. Ewing, J. Zhang, H. Xu, Y. Wang, G. M. Pauletti, D. B. Mast, D. Shi, Effect of spatial confinement on magnetic hyperthermia via dipolar interactions in $\mathrm{Fe}_{3} \mathrm{O}_{4}$ nanoparticles for biomedical applications, Mater. Sci. Eng. C 42 (2014) $52-63$.

[36] P. Guardia, N. Pérez, A. Labarta, X. Batlle, Controlled synthesis of iron oxide nanoparticles over a wide size range, Langmuir 26 (2010) 5843-5847.

[37] R. Skomski, X.-h. Wei, D. J. Sellmyer, Magnetization reversal in cubic nanoparticles with uniaxial surface anisotropy, IEEE Trans. Magn. 43 (2007) 2890-2892.

[38] A. Aharoni, J. P. Jakubovics, Cylindrical domains in small ferromagnetic spheres with cubic anisotropy, IEEE Trans. Magn. 24 (1988) 1892-1894.

[39] Q. Li, C. W. Kartikowati, S. Horie, T. Ogi, T. Iwaki, K. Okuyama, Correlation between particle size/domain structure and magnetic properties of highly crystalline $\mathrm{Fe}_{3} \mathrm{O}_{4}$ nanoparticles, Sci. Rep. 7 (2017) 9894.

[40] S. Disch, The spin structure of magnetic nanoparticles and in magnetic nanostructures, Ph.D. thesis, University of Aachen, Templergraben 55, 52062 Aachen, Germany, 2010. 
[54 C. Cheng, Structure and magnetic properties of the $\mathrm{Fe}_{3} \mathrm{O}_{4}(001)$ surface: Ab initio studies, Phys. Rev. B 71 (2005) 052401. loidal liquid crystals: Small-angle X-ray scattering studies with microradian resolution, Current Opinion in Colloid \& Interface Science 20 (2015) 272.

[42] S. H. Chen, J. Teixeira, Structure and fractal dimension of proteindetergent complexes, Phys. Rev. Lett. 57 (1986) 2583-2586.

[43] T. Freltoft, J. K. Kjems, S. K. Sinha, Power-law correlations and finite-size effects in silica particle aggregates studied by small-angle neutron scattering, Phys. Rev. B 33 (1986) 269-275.

[44] J. K. Percus, G. J. Yevick, Analysis of Classical Statistical Mechanics by Means of Collective Coordinates, Phys. Rev. 110 (1958) 1-13.

[45] D. J. Kinning, E. L. Thomas, Hard-Sphere Interactions Between Spherical Domains in Diblock Copolymers, Macromolecules 17 (1984) 1712-1718.

[46] M. B. Fernández Van Raap, P. Mendoza Zélis, D. F. Coral, T. E. Torres, C. Marquina, G. F. Goya, F. H. Sánchez, Self organization in oleic acidCoated $\mathrm{CoFe}_{2} \mathrm{O}_{4}$ colloids: A SAXS study, J. Nanoparticle Res. 14 (2012) 1072 .

[47] G. Singh et al., Self-assembly of magnetite nanocubes into helical superstructures, Science. 345 (2014) 1149-1153.

[48] S. Mehdizadeh Taheri, M. Michaelis, T.Friedrich, B. Förster, M. Drechsler, F. M. Römer, P. Bösecke, T. Narayanan, B. Weber, I. Rehberg, S. Rosenfeldt, S. Förster, Self-assembly of smallest magnetic particles, Proc. Natl. Acad. Sci. 112 (2015) 14484-14489.

[49] S. Sun, H. Zeng, D. B. Robinson, S. Raoux, P. M. Rice, S. X. Wang, G. Li, Monodisperse $\mathrm{MFe}_{2} \mathrm{O}_{4}(\mathrm{M}=\mathrm{Fe}, \mathrm{Co}, \mathrm{Mn})$ Nanoparticles, J. Am. Chem. Soc. 126 (2004) 273-279.

[50] S. Mørup, Magnetic hyperfine splitting in Mössbauer spectra of microcrystals, J. Magn. Magn. Mater, 37 (1983) 39-50.

[51] S. Mørup, H. Topsøe, Mössbauer studies of thermal excitations in magnetically ordered microcrystals, Appl. Phys. 11 (1976) 63-66.

[52] R. E. Vandenberghe, C. A. Barrero, G. M. da Costa, E. Van San, E. De Grave, Mössbauer characterization of iron oxides and (oxy)hydroxides: the present state of the art, Hyperfine Interact. 126 (2000) 247-259.

[53] G. M. Da Costa, E. De Grave, P. M. A. De Bakker, R. E. Vandenberghe, Influence of nonstoichiometry and the presence of maghemite on the Mössbauer spectrum of magnetite, Clays Clay Miner. 43 (1995) 656668 .

[41] A.V. Petukhov et al., Particle shape effects in colloidal crystals and col- 
[55] G.M. Da Costa et al., Magnetic Nanoparticles for in Vivo Use: A Critical Assessment of Their Composition, J. Phys. Chem. B 118(40) (2005) 11738.

[56] J. Fock et al., On the 'centre of gravity' method for measuring the composition of magnetite/maghemite mixtures, or the stoichiometry of magnetitemaghemite solid solutions, via ${ }^{57} \mathrm{Fe}$ Mssbauer spectroscopy, J. Phys. D: Appl. Phys. 50 (2017) 265005.

[57] J. P. Sheperd et al., Heat capacity and entropy of nonstoichiometric magnetite $\mathrm{Fe}_{3(1-\delta)} \mathrm{O}_{4}$ : the thermodynamic nature of the Verwey transition, Phys. Rev. B. 43 (1991) 8461.

[58] Z. Kakol, J. Sabol, J. M. Honig, Magnetic anisotropy of titanomagnetites $\mathrm{Fe}_{3-x} \mathrm{Ti}_{x} \mathrm{O}_{4}, 0 \leq x \leq 0.55$, Phys. Rev. B 44 (1991) 2198-2204.

[59] P. Ludwig et al., Size analysis of single-core magnetic nanoparticles, J. Magn. Magn. Mater. 427 (2017) 19-24.

[60] P. Bender et al., Distribution functions of magnetic nanoparticles determined by a numerical inversion method, New. J. Phys. 19 (2017) 073012.

[61] S. Sakurai, N. Soda, M. Kobayashi, G. Rowlands, Geometries with the demagnetizing energy independent of the direction of magnetization, IEEE Trans. Magn. 43 (2007) 982-991.

[62] G. Muscas, G. Concas, C. Cannas, A. Musinu, A. Ardu, F. Orrù, D. Fiorani, S. Laureti, D. Rinaldi, G. Piccaluga, D. Peddis, Magnetic properties of small magnetite nanocrystals, J. Phys. Chem. C 117 (2013) 23378-23384.

[63] K. Lee, J.-t. Jang, H. Nakano, S. Nakagawa, S. H. Paek, S. Bae, External magnetic field dependent shift of superparamagnetic blocking temperature due to core/surface disordered spin interactions, Nanotechnology 28 (2017) 075710 .

[64] E. Lima, A. L. Brandl, A. D. Arelaro, G. F. Goya, Spin disorder and magnetic anisotropy in $\mathrm{Fe}_{3} \mathrm{O}_{4}$ nanoparticles, J. Appl. Phys. 99 (2006).

[65] A. T. Ngo, P. Bonville, M.P. Pileni, Nanoparticles of $\mathrm{Co}_{x} \mathrm{Fe}_{y} \square_{z} \mathrm{O}_{4}$ : Synthesis and superparamagnetic properties, Eur. Phys. J. B 592 (1999) 583-592.

[66] C. R. Vestal, Z. J. Zhang, Effects of surface coordination chemistry on the magnetic properties of $\mathrm{MnFe}_{2} \mathrm{O}_{4}$ spinel ferrite nanoparticles, J. Am. Chem. Soc. 125 (2003) 9828-9833.

[67] T. J. Daou, J. M. Grenèche, G. Pourroy, S. Buathong, A. Derory, C. UlhaqBouillet, B. Donnio, D. Guillon, S. Begin-Colin, Coupling Agent Effect on Magnetic Properties of Functionalized Magnetite-Based Nanoparticles, Chem Mater. 20 (2008) 5869-5875.

[68] E. J. Verwey, P. W. Haayman, Electronic conductivity and transition point of magnetite $\mathrm{Fe}_{3} \mathrm{O}_{4}$, Phys. Rev. B 8 (1941) 979-987. 
[69] R. Aragan, D. J. Buttrey, J. P. Shepherd, J. M. Honig, Influence of nonstoichiometry on the Verwey transition, Phys. Rev. B 31 (1985) 430-436.

[70] G. F. Goya, T. S. Berquó, F. C. Fonseca, M. P. Morales, Static and dynamic magnetic properties of spherical magnetite nanoparticles, J. Appl. Phys. 94 (2003) 3520-3528.

[71] J. Santoyo Salazar, L. Perez, O. De Abril, L. Truong Phuoc, D. Ihiawakrim, M. Vazquez, J. M. Greneche, S. Begin-Colin, G. Pourroy, Magnetic iron oxide nanoparticles in 10-40 $\mathrm{nm}$ range: Composition in terms of magnetite/maghemite ratio and effect on the magnetic properties, Chem. Mater. 23 (2011) 1379-1386.

[72] R. Das, J. Alonso, Z. Nemati Porshokouh, V. Kalappattil, D. Torres, M. H. Phan, E. Garaio, J. Á. García, J. L. Sanchez Llamazares, H. Srikanth, Tunable High Aspect Ratio Iron Oxide Nanorods for Enhanced Hyperthermia, J. Phys. Chem. C 120 (2016) 10086-10093.

[73] B. D. Cullity, C. D. Graham, Introduction to Magnetic Materials, AddisonWesley Publishing Co.,1972.

[74] W. Baaziz, B. P. Pichon, S. Fleutot, Y. Liu, C. Lefevre, J. M. Greneche, M. Toumi, T. Mhiri, S. Begin-Colin, Magnetic irón oxide nanoparticles: Reproducible tuning of the size and nanosized-dependent composition, defects, and spin canting, J. Phys. Chem. C 118 (2014) 3795-3810.

[75] M. E. Materia, P. Guardia, A. Sathya, M. Pernia Leal, R. Marotta, R. Di Corato, T. Pellegrino, Mesoscale assemblies of iron oxide nanocubes as heat mediators and image contrast agents, Langmuir 31 (2015) 808-816.

[76] Z. Nemati, J. Alonso, L. M. Martinez, H. Khurshid, E. Garaio, J. A. Garcia, M. H. Phan, H. Srikanth, Enhanced Magnetic Hyperthermia in Iron Oxide Nano-Octopods: Size and Anisotropy Effects, J. Phys. Chem. C 120 (2016) 8370-8379.

[77] P. Guardia, R. Di Corato, L. Lartigue, C. Wilhelm, A. Espinosa, M. GarciaHernandez, F. Gazeau, L. Manna, T. Pellegrino, Water-soluble iron oxide nanocubes with high values of specific absorption rate for cancer cell hyperthermia treatment, ACS Nano 6 (2012) 3080-3091.

[78] D. F. Coral, P. Mendoza Zélis, M. Marciello, M. D. P. Morales, A. Craievich, F. H. Sánchez, M. B. Fernández Van Raap, Effect of Nanoclustering and Dipolar Interactions in Heat Generation for Magnetic Hyperthermia, Langmuir 32 (2016) 1201-1213.

[79] D. Cabrera, A. Coene, J. Leliaert, E. J. Arts-Ibez, L. Dupr, N. D. Telling, F. J. Teran, Dynamical Magnetic Response of Iron Oxide Nanoparticles Inside Live Cells, ACS Nano 12 (2018) 2741-2572. 
[80] I. J. Bruvera et al., Typical experiment vs. in-cell like conditions in magnetic hyperthermia: Effects of media viscosity and agglomeration, J. Magn. Magn. Mater. 491 (2019) 115563. 